\title{
BMJ Open Diagnostic accuracy of screening tests for COPD: a systematic review and meta-analysis
}

\author{
Shamil Haroon, Rachel Jordan, Yemisi Takwoingi, Peymane Adab
}

To cite: Haroon S, Jordan R, Takwoingi $\mathrm{Y}$, et al. Diagnostic accuracy of screening tests for COPD: a systematic review and meta-analysis. BMJ Open 2015;5:e008133. doi:10.1136/bmjopen-2015008133

- Prepublication history and additional material is available. To view please visit the journal (http://dx.doi.org/ 10.1136/bmjopen-2015008133).

Received 15 March 2015 Revised 3 September 2015 Accepted 11 September 2015

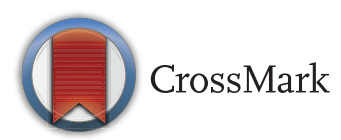

Public Health, Epidemiology \& Biostatistics, School of Health and Population Sciences, University of Birmingham, Birmingham, UK

Correspondence to Dr Rachel Jordan; r.e.jordan@bham.ac.uk

\section{ABSTRACT}

Background: Chronic obstructive pulmonary disease (COPD) is widely underdiagnosed. A number of studies have evaluated the accuracy of screening tests for COPD, but their findings have not been formally summarised. We therefore sought to determine and compare the diagnostic accuracy of such screening tests in primary care.

Methods: Systematic review and meta-analysis of the diagnostic accuracy of screening tests for COPD confirmed by spirometry in primary care. We searched MEDLINE, EMBASE and other bibliographic databases from 1997 to 2013 for diagnostic accuracy studies that evaluated 1 or more index tests in primary care among individuals aged $\geq 35$ years with no prior diagnosis of COPD. Bivariate meta-analysis of sensitivity and specificity was performed where appropriate.

Methodological quality was assessed independently by 2 reviewers using the QUADAS- 2 tool.

Results: 10 studies were included. 8 assessed screening questionnaires (the COPD Diagnostic Questionnaire (CDQ) was the most evaluated, $n=4), 4$ assessed handheld flow meters (eg, COPD-6) and 1 assessed their combination. Among ever smokers, the CDQ (score threshold $\geq 19.5 ; n=4$ ) had a pooled sensitivity of $64.5 \%(95 \% \mathrm{Cl} 59.9 \%$ to $68.8 \%)$ and specificity of $65.2 \%$ (52.9\% to $75.8 \%)$, and handheld flow meters $(n=3)$ had a sensitivity of $79.9 \%(95 \% \mathrm{Cl}$ $74.2 \%$ to $84.7 \%)$ and specificity of $84.4 \%(68.9 \%$ to $93.0 \%)$. Inadequate blinding between index tests and spirometry was the main risk of bias.

Conclusions: Handheld flow meters demonstrated higher test accuracy than the CDQ for COPD screening in primary care. The choice of alternative screening tests within whole screening programmes should now be fully evaluated.

PROSPERO registration number: CRD42012002074.

\section{INTRODUCTION}

Chronic obstructive pulmonary disease (COPD) is the third leading cause of death, ${ }^{1}$ ranks ninth for lost disability adjusted life years, ${ }^{2}$ and is an important cause of healthcare expenditure. ${ }^{3}$ Despite this, as much as $50-90 \%$ of the disease burden remains undiagnosed. ${ }^{4}$

\section{Strengths and limitations of this study}

- This is the first systematic review and meta-analysis of the diagnostic accuracy of screening tests for chronic obstructive pulmonary disease (COPD) in primary care.

- Robust methods were used to identify, appraise and summarise the available literature.

- There were few head-to-head comparisons of screening tests.

- The definition of COPD used in the majority of included studies was physiological, based on the presence of airflow limitation, rather than clinical, requiring the presence of relevant symptoms.

- Methodological limitations of included studies included inadequate reporting of blinding of operators performing and interpreting screening and reference tests (spirometry) and reporting of withdrawals and indeterminate results.

Patients often under-recognise the significance of respiratory symptoms, ${ }^{5}$ and clinicians frequently miss opportunities to diagnose COPD at primary care consultations. ${ }^{6}$ Early detection may offer opportunities to reduce disease progression and improve quality of life, for example, through smoking cessation interventions $^{7}$ and pulmonary rehabilitation. ${ }^{8}$ An analysis of the Health Survey for England suggested that over three-quarters of symptomatic smokers identified with COPD through targeted case finding could benefit from recommended therapies, which could potentially prevent hospitalisations. ${ }^{9}$

There is now a policy drive to identify undiagnosed COPD. ${ }^{10}{ }^{11}$ However, a systematic review of population-based screening with spirometry concluded that this should not be recommended, partly because it estimated that hundreds of smokers would need to be screened to prevent a single COPD exacerbation. ${ }^{12}$ Furthermore, without considering clinical symptoms, this approach could identify individuals with airflow obstruction, who would not meet the clinical criteria for COPD according to current guidelines. ${ }^{11}$ 
Recently, efforts to identify undiagnosed COPD have focused on the use of initial screening tests to identify those at high risk, prior to diagnostic spirometry. ${ }^{13} 14$ Several approaches for initial screening have been evaluated, but their findings have not been systematically reviewed and quantitatively synthesised, and it is not yet clear which test or combination is the most accurate. Although one narrative review ${ }^{15}$ compared existing symptom-based questionnaires, it did not include other screening tests and needs updating.

We report a systematic review and meta-analysis of published studies that summarises and compares the accuracy of screening tests for COPD in primary care.

\section{METHODS}

Protocol and registration

The protocol for this review was previously published ${ }^{16}$ and registered. ${ }^{17}$

\section{Eligibility criteria}

We sought diagnostic accuracy studies of any design that evaluated one or more index tests, were conducted in primary care (including general practices and community pharmacies) and recruited individuals aged $\geq 35$ years with no prior diagnosis of COPD. Index tests included screening questionnaires, handheld flow meters (eg, Piko-6 or COPD-6), peak flow meters, chest radiography, and risk prediction models or decision aids, either alone or in combination. We only included studies that specified the target condition as COPD, and used the presence of airflow obstruction, based on prebronchodilator or postbronchodilator spirometry as the reference standard (although postbronchodilator spirometry was considered the ideal reference standard).

\section{Outcomes}

The primary outcome was identification of COPD. The main measures of test accuracy examined were sensitivity and specificity.

\section{Search strategy}

We searched the following databases from March/April 2012 for the previous 15 years: MEDLINE, EMBASE, CINAHL, Cochrane Central Register of Controlled Trials and the Health Technology Database. We also performed an updated search on MEDLINE and EMBASE up to December 2013. Searches limited to the first 100 articles were also performed on Google Scholar, Turning Research into Practice, HTAi VORTAL and DogPile, and selected conference abstracts for the previous 2 years. Search terms are listed in online supplementary table S1 and included Medical Subject Heading terms and free-text synonyms for COPD, screening tests and measures of test accuracy, with no language restrictions.

\section{Study selection and data extraction}

Titles and abstracts were screened independently by two reviewers. Relevant full-text articles were independently assessed for eligibility by two reviewers and disagreements resolved through discussion. Prespecified data were extracted from full-text articles by one reviewer and verified by a second. We extracted the number of true positives, false positives, true negatives and false negatives for construction of two-by-two contingency tables. Where these data were not provided, reported measures of test accuracy were used to derive these values.

\section{Risk of bias assessment}

Included studies were assessed independently by two reviewers for risk of methodological bias and applicability concerns against criteria from the QUADAS-2 tool. ${ }^{18}$ Online supplementary table S2 shows how this was adapted for the review. Disagreements were resolved through discussion.

\section{Statistical analysis}

Forest plots of sensitivity and specificity were constructed using Review Manager (RevMan) V.5.2 (Copenhagen: The Nordic Cochrane Centre, The Cochrane Collaboration, 2012). These plots were used to visually explore between-study variation in the diagnostic accuracy of each test. We also explored differences in population screened, screening test, diagnostic criteria and study design.

Where there was sufficient clinical and methodological homogeneity, we used the xtmelogit command in Stata V.13.1 (Stata-Corp, College Station, Texas, USA) to fit the bivariate model ${ }^{19}{ }^{20}$ to derive summary estimates of sensitivity and specificity and their $95 \%$ CIs. If there were fewer than four studies, we simplified the bivariate model to two univariate random effects logistic regression models for sensitivity and specificity by assuming no correlation between both measures. ${ }^{21}$ We used two approaches to compare the diagnostic accuracy of the screening tests. First, we used all relevant studies that evaluated one or more tests, and second we restricted the analysis to studies that made direct (head-to-head) comparisons. Where meta-analysis was possible, tests were compared by adding a covariate for test type to the bivariate model to assess whether average sensitivity and/or specificity differed between the tests. To assess the statistical significance of differences in sensitivity and specificity between tests, we compared the fit of alternative models (effect of adding or removing covariate terms from the model) by using likelihood ratio tests.

Positive and negative predictive values (PPV and NPV) were estimated from the sensitivity and specificity of each test, assuming a prevalence of undiagnosed COPD of $5.5 \%{ }^{9}$ in a hypothetical population of 1000 patients aged $\geq 40$ years. We estimated the number-needed-toscreen to identify one individual with COPD as the total number screened divided by the number of true 
positives, and the number of diagnostic assessments needed as the reciprocal of the PPV.

\section{RESULTS}

\section{Study selection}

The stages of study selection are shown in figure 1. After excluding duplicates, our search yielded 2605 records. From these, full-text articles were retrieved for 266 studies. Ten studies met the inclusion criteria, and five were suitable for meta-analysis (since these were sufficiently similar with respect to the included population, screening tests and definition of COPD). Figure 1 lists the reasons for excluding articles, the most common of which was the inclusion of patients with previously known COPD.

\section{Study characteristics}

Characteristics of included studies are summarised in tables 1 and 2 (see online supplementary tables S3-5 for details of each study). All were cross-sectional test accuracy studies, of which two used a paired design to compare two screening tests (screening questionnaires and handheld flow meters). ${ }^{22}{ }^{23}$ Nine studies were multicentre and all were based in general practices.

\section{Recruitment and population selection}

Four studies opportunistically recruited patients routinely attending primary care, three actively recruited participants through postal invitations or local advertisements, two used a combination of both strategies and one study did not report the method of recruitment. ${ }^{24}$ All studies specified age in the inclusion criteria with most requiring participants to be over 40 years. Seven studies also required a positive smoking history, but only one required participants to report respiratory symptoms as part of the entry criteria. ${ }^{13}$ The main exclusion criterion was an established history of lung disease.

\section{Index and reference tests}

All studies first applied one or more index tests to the eligible population and then performed the reference test (spirometry) on either all ( $\mathrm{n}=8$ studies) or a random sample ${ }^{25} 26(\mathrm{n}=2)$ of participants. Index tests included screening questions or questionnaires $(n=8)$ and handheld flow meters $(n=4)$. One study also assessed the combined accuracy of using a screening questionnaire sequentially with a handheld flow meter. ${ }^{22}$ No studies evaluating other screening tests met the inclusion criteria.

\section{Reference standard}

Prebronchodilator and postbronchodilator spirometry was the reference standard in two ${ }^{25} 27$ and eight studies, respectively (tables 1 and 2). Most studies sufficiently described spirometry and quality control procedures. Spirometry was performed by trained technicians $(n=4)$,
Figure 1 Study selection (COPD, chronic obstructive pulmonary disease).

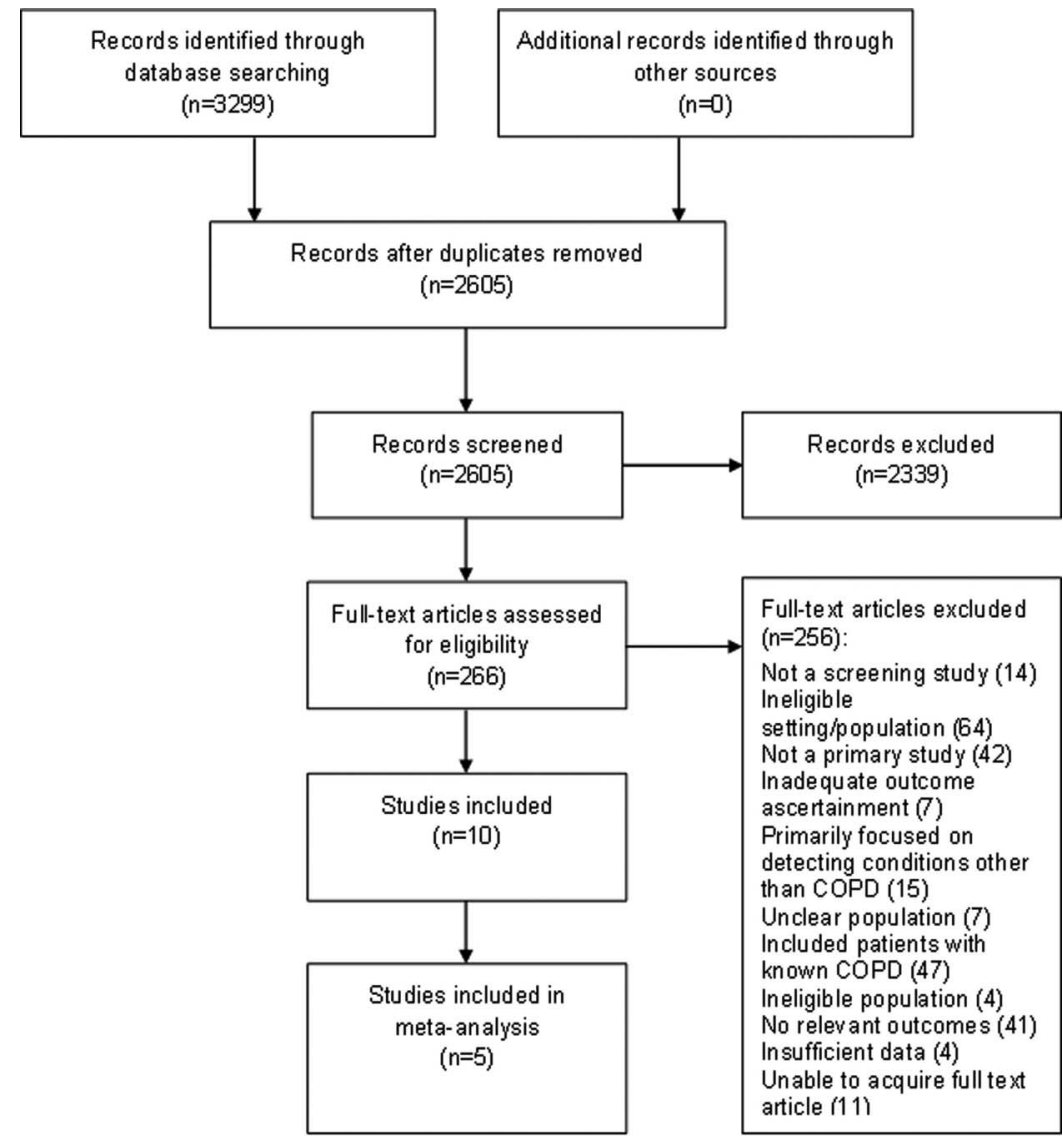


Table 1 Characteristics of studies evaluating screening questionnaires (8 studies) ${ }^{1322-25272838}$

\section{Characteristic}

Study designs

Participants

Mean age (years)

Male (\%)

Required smoking status

Required respiratory symptoms

Setting

Number of centres

Recruitment strategy

Questionnaires

Common items

\section{Reference test-spirometry}

Post-BD

Definition of airflow obstruction

Included symptoms in definition of COPD

Spirometry quality control

Range of results

Sensitivity

Specificity

Severity of new COPD cases

$\left(\mathrm{FEV}_{1} \%\right.$ predicted)‡
Cross-sectional test accuracy

Only current/ex-smokers

Included never-smokers

General practice(s)

Multicentre

Single centre

Active

Opportunistic

Active and opportunistic

Not reported

COPD Diagnostic Questionnaire*

Lung Function Questionnaire

Not named

Age

Smoking status

Respiratory symptoms

Allergies

Range/number of studies

8

237-3158

$52.3-65.3$

$38.1-69.0$

5

3

$1-36$

7

1

1

7

7

8

5

Post-BD FEV $/$ FVC $<0.7$

Other†

Yes

$57-93 \%$

$24-80 \%$

$11-39 \%$

$\geq 80 \%$

$50-80 \%$

$43-61 \%$

$<50 \%$

${ }^{*}$ Also referred to as the Respiratory Health Screening Questionnaire and the IPAG questionnaire.

†Pre-BD FEV $/$ FVC $<88.5 \%$ predicted for men and $\mathrm{FEV}_{1} / \mathrm{FVC}<89.3 \%$ for women.

łBased on five studies.

$\mathrm{BD}$, bronchodilator; COPD, chronic obstructive pulmonary disease; FEV ${ }_{1}$, forced expiratory volume in 1 s; FVC, forced vital capacity; IPAG,

International Primary Airways Group.

general practitioners (GPs; $\mathrm{n}=1)$, pulmonary physicians $(\mathrm{n}=1)$ and nurses $(\mathrm{n}=2)$, while quality control was usually performed by a respiratory specialist or physiologist who reviewed spirometry results.

\section{Methodological quality}

Most studies gave a clear description of participants, index and reference tests, and diagnostic criteria (see online supplementary figure S1 and table S6). However, there was often under-reporting of withdrawals $(n=4)$, participant flow diagrams $(n=5)$ and uninterpretable spirometry tests $(n=5)$. The main risk of bias arose from inadequate blinding between index and reference tests $(\mathrm{n}=7$; figures 2 and 3). There was also potential for bias in the flow and timing domain $(n=5)$, where the number of participants undergoing index and reference tests was unclear, and where significant numbers of participants were excluded from the analysis.

\section{Screening questionnaires}

Altogether four screening questionnaires were evaluated on a total of 9472 participants in eight studies (table 1), of which the COPD Diagnostic Questionnaire (CDQ) ${ }^{28}$ also referred to as the International Primary Airways Group (IPAG) Questionnaire, ${ }^{22}$ was the most widely validated $(\mathrm{n}=4) .{ }^{13}{ }^{22} 2328$ All instruments included questions related to the presence of respiratory symptoms (usually cough, dyspnoea and wheeze). Other items included in some, but not all questionnaires related to smoking history, allergies, age, body mass index (BMI) 
Table 2 Characteristics of studies evaluating handheld flow meters (4 studies) 14222326

\begin{tabular}{|c|c|c|}
\hline Characteristic & & Range/number of studies \\
\hline Study designs & Cross-sectional test accuracy study & 4 \\
\hline Participants & & $305-2464$ \\
\hline Mean age (years) & & $52.0-65.3$ \\
\hline Male (\%) & & 43.3-99.7 \\
\hline \multirow[t]{2}{*}{ Required smoking status } & Only current/ex-smokers & 3 \\
\hline & Included never-smokers & 1 \\
\hline Required respiratory symptoms & & 0 \\
\hline Setting & General practice(s) & 4 \\
\hline \multirow[t]{2}{*}{ Number of centres } & & $4-25$ \\
\hline & Multicentre & 4 \\
\hline \multirow[t]{3}{*}{ Recruitment strategy } & Active & 1 \\
\hline & Opportunistic & 2 \\
\hline & Active and opportunistic & 1 \\
\hline \multicolumn{3}{|l|}{ Handheld flow meter } \\
\hline \multirow[t]{2}{*}{ Device } & Piko-6 & 3 \\
\hline & COPD-6 & 1 \\
\hline \multirow[t]{3}{*}{ Operator } & Nurse & 2 \\
\hline & GP & 1 \\
\hline & Not reported & 1 \\
\hline \multirow[t]{2}{*}{ Use of BD } & Pre-BD & 3 \\
\hline & Post-BD & 1 \\
\hline Test threshold & $\mathrm{FEV}_{1} / \mathrm{FEV}_{6}<$ & $0.70-0.75$ \\
\hline \multicolumn{3}{|l|}{ Reference test-spirometry } \\
\hline Post-BD & & 4 \\
\hline Definition of airflow obstruction & Post-BD FEV $1 / F V C<0.7$ & 4 \\
\hline Included symptoms in definition of COPD & & 0 \\
\hline \multirow[t]{3}{*}{ Spirometry quality control } & Yes & 2 \\
\hline & No & 1 \\
\hline & Unclear & 1 \\
\hline \multicolumn{3}{|l|}{ Range of results } \\
\hline Sensitivity & & $79-86 \%$ \\
\hline Specificity & & $71-99 \%$ \\
\hline Severity of new COPD cases & $\geq 80 \%$ & $35-48 \%$ \\
\hline \multirow{2}{*}{$\left(\mathrm{FEV}_{1} \%\right.$ predicted $^{3}$} & $50-80 \%$ & $48-65 \%$ \\
\hline & $<50 \%$ & $0-16 \%$ \\
\hline
\end{tabular}

$\mathrm{BD}$, bronchodilator; COPD, chronic obstructive pulmonary disease; $\mathrm{FEV}_{1}$, forced expiratory volume in $1 \mathrm{~s}$; FVC, forced vital capacity; GP, general practitioner.

and physical functioning. Overall, participants were similar in age (range 52.3-65.3 years) but varied by sex (range $38-69 \%$ male).

\section{COPD Diagnostic Questionnaire}

Four studies ${ }^{13} 222328$ that evaluated the CDQ in ever smokers were included in a meta-analysis. Using a score threshold of $\geq 19.5$, the pooled sensitivity was $64.5 \%$ (95\% CI $59.9 \%$ to $68.8 \%)$ and specificity $65.2 \%(95 \%$ CI $52.9 \%$ to $75.8 \%$; table 3$)$. With a prevalence of undiagnosed COPD of $5.5 \%$, this gave a PPV of $9.7 \%$ (95\% CI $6.9 \%$ to $14.2 \%$ ), NPV of $96.9 \%$ (95\% CI $95.8 \%$ to $97.7 \%$ ), and would require 29 individuals (95\% CI 27 to 31$)$ to complete the CDQ and $11(95 \%$ CI 7 to 15$)$ to undergo a diagnostic assessment to identify one individual with COPD. At a lower score threshold of $\geq 16.5$, the pooled sensitivity was higher but the specificity lower, requiring 21 individuals (95\% CI 20 to 22) to complete the questionnaire and 13 (95\% CI 11 to 16$)$ to undergo a diagnostic assessment for each new diagnosis.

\section{All other questionnaires}

There was considerable between-study heterogeneity in the design of other screening questionnaires, which precluded their meta-analysis. In these four studies, sensitivities ranged from $57 \%$ to $88 \%$ and specificities from $25 \%$ to $80 \%$ (figure 4 ).

\section{Handheld flow meters}

The test accuracy of handheld flow meters was evaluated in 1400 participants across four studies (table 2). ${ }^{14} 222326$ Participants were similar in age (range 52-65.3 years) but varied by sex (range 43-99.7\% male). Only one study included never-smokers and stratified the results by smoking status. ${ }^{22}$ 
Figure 2 Risk of bias and applicability concerns graph: review authors' judgements about each domain presented as percentages across included studies.

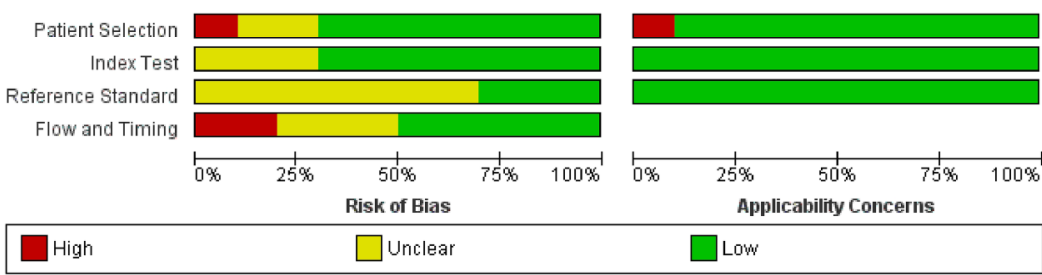

Handheld flow meters differ from diagnostic spirometers in that they are limited to measuring the forced expiratory volume in 1 and $6 \mathrm{~s}\left(\mathrm{FEV}_{1}\right.$ and $\mathrm{FEV}_{6}$, respectively), are usually performed with three blows and are cheaper and quicker to administer. They were used without a bronchodilator in three studies ${ }^{14} 2326$ and were supervised by either trained nurses or GPs. A narrow range of thresholds were used to denote a positive test ranging from $\mathrm{FEV}_{1} / \mathrm{FEV}_{6}<0.7$ to 0.75 .

Their sensitivity ranged from $79 \%$ to $86 \%$ and specificity from $71 \%$ to $99 \%$ (figure 4). Three studies ${ }^{1422} 23$ enrolling ever smokers were similar enough to be included in a meta-analysis. The pooled sensitivity was $79.9 \%$ (95\% CI $74.2 \%$ to $84.7 \%$ ) and specificity was $84.4 \%$ (95\% CI $68.9 \%$ to $93.0 \%)$. Using the same assumptions, this would require 23 individuals (95\% CI 22 to 24 ) to be screened and 5 (95\% CI 3 to 9) to undergo a diagnostic assessment to identify 1 individual with COPD (table 3).

\section{Combination of tests}

In the single study that reported the combined accuracy of a screening questionnaire (CDQ) with a handheld flow meter, the sensitivity was $74 \%(95 \%$ CI $64 \%$ to

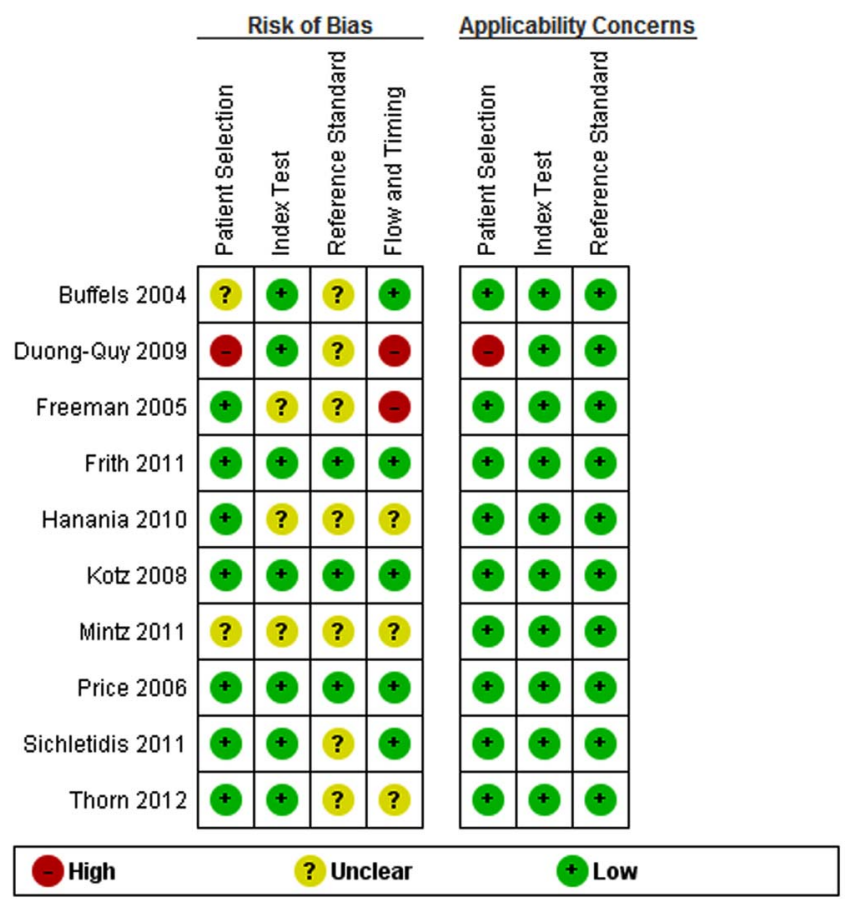

Figure 3 Risk of bias and applicability concerns summary: review authors' judgements about each domain for each included study.
$83 \%$ ) and specificity was $97 \%$ (95\% CI $95 \%$ to $98 \%) .{ }^{22}$ This would reduce the need for diagnostic assessment to two individuals (95\% CI 2 to 3 ) to identify one with COPD (table 3 and figure 5).

\section{Comparison of test accuracy}

In the first comparative analysis, based on an indirect comparison in ever smokers, there was evidence from the likelihood ratio tests that the $\mathrm{CDQ}$ at a score threshold of $\geq 19.5$ had a lower sensitivity $(\mathrm{p}=0.003)$ but no difference in specificity $(\mathrm{p}=0.09$ ) compared with handheld flow meters. In the second analysis at the lower score threshold of $\geq 16.5$ (or 17), there was evidence to suggest a higher sensitivity $(\mathrm{p}=0.03)$ but a much lower specificity $(\mathrm{p}=0.01)$ than handheld flow meters. Two studies directly compared handheld flow meters and the $\mathrm{CDQ}^{22}{ }^{23}$ and their findings were consistent with the results of the indirect comparison. Furthermore, Frith et $a t^{23}$ also reported both higher sensitivity and specificity of handheld flow meters compared with the CDQ at the score threshold of $\geq 19.5$.

\section{DISCUSSION}

\section{Summary of evidence}

This review incorporated evidence on the test accuracy of questionnaires and handheld flow meters for COPD screening in primary care. The CDQ developed by Price $e t a l^{28}$ was the most widely validated of the four screening questionnaires included. However, use of handheld flow meters under the supervision of trained health professionals was significantly more accurate than the $\mathrm{CDQ}$ for discriminating between ever smokers with and without airway obstruction, and a combination of both instruments may improve the accuracy still further, potentially reducing the number of diagnostic assessments required. ${ }^{22}$ Studies evaluating the CDQ and handheld flow meters had generally few methodological biases, the main being insufficient clarity on blinding between index and reference tests.

Unfortunately, only one study by Kotz et $a l^{13}$ considered the accuracy of a screening test (handheld flow meter) for identifying airflow obstruction in symptomatic patients, which is closer to identifying clinical COPD. The remainder evaluated the accuracy for identifying airflow obstruction without explicitly considering the presence of symptoms. Nevertheless, the results are still likely to apply since we observed that the test accuracy reported by Kotz et $a l^{13}$ was very similar to that reported by studies that did not explicitly consider respiratory symptoms. 


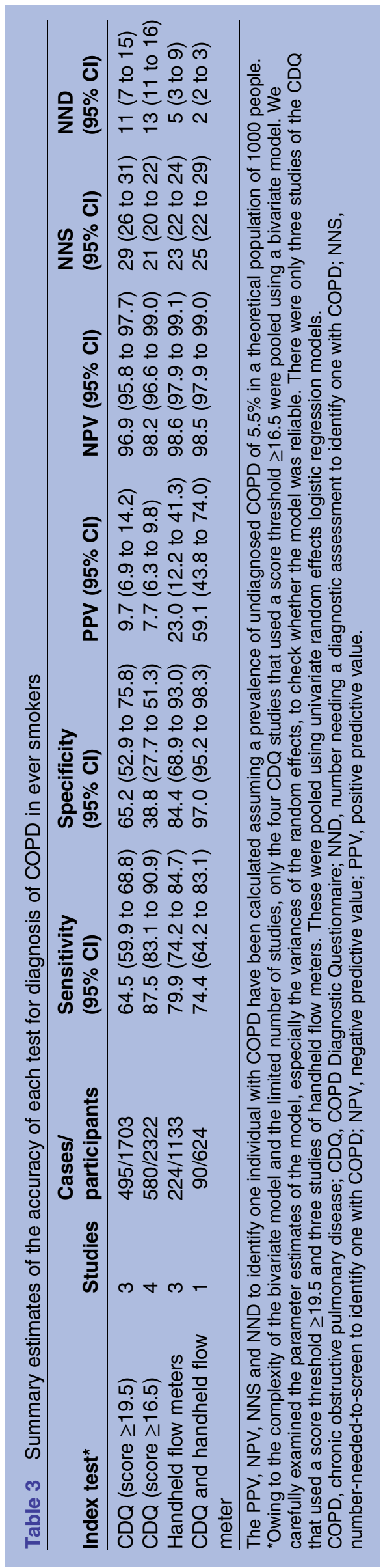

Relationship to other studies

The US Preventive Services Task Force (USPSTF) and the UK National Screening Committee recommended against routine screening for COPD partly due to concerns about efficiency and costs. ${ }^{12} 29$ However, the USPSTF evidence review did not consider the use of screening tests such as questionnaires and handheld flow meters that may help triage high-risk patients for diagnostic assessment as suggested by our findings. Screening was recommended against on the basis that it would lead largely to the diagnosis of mild-to-moderate disease, for which there is limited evidence on effective interventions. ${ }^{12} 30$ However, a significant proportion of new diagnoses of COPD in our included studies had moderate-to-severe airflow obstruction $\left(48.9 \%^{13}\right.$ to $88.5 \%^{27}$ with an $\mathrm{FEV}_{1}$ $<80 \%$ predicted)-these patients are likely to benefit from established therapies for COPD. ${ }^{8}$

In 2005, van Schayck et $a l^{15}$ compared symptom-based questions for identifying COPD and validated their accuracy using data from the National Health and Nutrition Examination Survey (NHANES) III. Age, BMI, smoking status, smoking intensity, self-reported asthma, chronic bronchitis or emphysema, and chronic cough or phlegm represented the optimal combination of variables for identifying individuals with airflow obstruction, having a sensitivity of $71 \%$ and specificity of $67 \%$. Many of these risk factors have been incorporated in screening questionnaires evaluated in our review and their combined accuracy appears to be lower than handheld flow meters. Furthermore, a meta-analysis of studies evaluating the accuracy of $\mathrm{FEV}_{1} / \mathrm{FEV}_{6}$ measured by standard diagnostic spirometry for detecting airflow obstruction showed it has a sensitivity of 0.89 (95\% CI 0.83 to 0.93 ) and specificity of $0.98(95 \% 0.95$ to 0.99$) .{ }^{31}$ While the accuracy of handheld flow meters (which measure $\mathrm{FEV}_{1} / \mathrm{FEV}_{6}$ ) appears to be lower than this, the findings from the current review suggest that they are still sufficiently accurate to screen for airflow limitation.

Finally, we identified two recent relevant studies that fell outside the time window of our literature search. The first invited ever smokers aged 40-85 years from 36 general practices to complete the $\mathrm{CDQ}$ and perform spirometry. ${ }^{32}$ The CDQ showed a sensitivity and specificity of $63.0 \%$ and $70.1 \%$, respectively, when using a score threshold of $\geq 19.5$ and $79.7 \%$ and $46.8 \%$ using a cut-point of $\geq 16.5$. The second study evaluated the NPV of handheld flow meters among a small sample $(n=54)$ of ex-smokers aged $\geq 50$ years who had been referred for diagnostic spirometry by their GP. ${ }^{33}$ The NPV was estimated at $94.4 \%$ (95\% CI $86.4 \%$ to $98.5 \%$ ) when using the fixed ratio of $\mathrm{FEV}_{1} /$ forced vital capacity $(\mathrm{FVC})<0.7$ to define airflow obstruction. Both findings are in keeping with our meta-analyses.

\section{Strengths and weaknesses of the review}

Strengths of this review include the methods used to identify and appraise the available literature. Other than the limitation of the case definition discussed above, the 
COPD Diagnostic Questionnaire (score threshold $\geq 19.5$ )

$\begin{array}{lrrrrrr}\text { Study } & \text { TP } & \text { FP } & \text { FN } & \text { TN } & \text { Sensitivity }(95 \% \text { Cl) } & \text { Specificity }(95 \% \text { Cl) } \\ \text { Frith 2011 } & 45 & 56 & 17 & 91 & 0.73[0.60,0.83] & 0.62[0.54,0.70] \\ \text { Kotr 2008 } & 183 & 183 & 95 & 215 & 0.66[0.60,0.71] & 0.54[0.49,0.59] \\ \text { Price 2006 } & 91 & 152 & 64 & 511 & 0.59[0.51,0.67] & 0.77[0.74,0.80]\end{array}$

COPD Diagnostic Questionnaire (score threshold $\geq 16.5$ or $\geq 17$ )
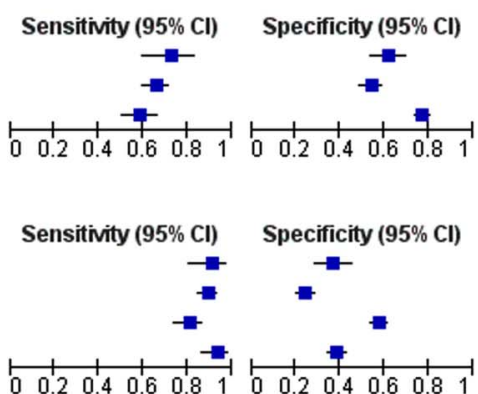

All other screening questionnaires

$\begin{array}{lrrrrrr}\text { Stucly } & \text { TP } & \text { FP } & \text { FN } & \text { TN } & \text { Sensitivity }(95 \% \text { Cl) } & \text { Specificity (95\% Cl) } \\ \text { Buffels 2004 } & 130 & 598 & 99 & 2331 & 0.57[0.50,0.63] & 0.80[0.78,0.81] \\ \text { Freeman 2005 } & 54 & 88 & 8 & 219 & 0.87[0.76,0.94] & 0.71[0.66,0.76] \\ \text { Freeman } 2005^{2} & 48 & 73 & 14 & 234 & 0.77[0.65,0.87] & 0.76[0.71,0.81] \\ \text { Hanania 2010 } & 129 & 355 & 27 & 326 & 0.83[0.76,0.88] & 0.48[0.44,0.52] \\ \text { Mintz 2011 } & 143 & 413 & 19 & 138 & 0.88[0.82,0.93] & 0.25[0.21,0.29]\end{array}$

Handheld spirometry

$\begin{array}{lrrrrrr}\text { Study } & \text { TP } & \text { FP } & \text { FN } & \text { TN } & \text { Sensitivity }(95 \% \text { Cl) } & \text { Specificity }(95 \% \text { Cl) } \\ \text { Duong-Quy 2009 } & 306 & 18 & 50 & 2023 & 0.86[0.82,0.89] & 0.99[0.99,0.99] \\ \text { Frith 2011 } & 46 & 43 & 11 & 104 & 0.81[0.68,0.90] & 0.71[0.63,0.78] \\ \text { Sichletidis 2011 } & 72 & 32 & 18 & 502 & 0.80[0.70,0.88] & 0.94[0.92,0.96] \\ \text { Thorn 2012 } & 61 & 45 & 16 & 183 & 0.79[0.68,0.88] & 0.80[0.74,0.85]\end{array}$

COPD Diagnostic Questionnaire \& handheld spirometer
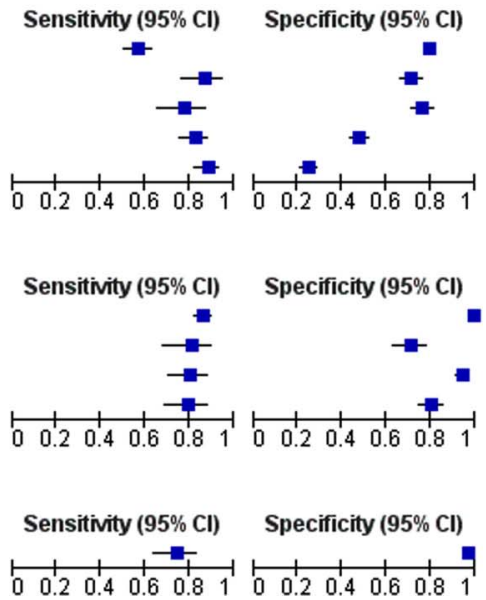

Figure 4 Forest plot of sensitivity and specificity of each screening test. (1) Binary response questionnaire, (2) multiple response questionnaire. COPD, chronic obstructive pulmonary disease; TP, true positive; FP, false positive; FN, false negative; TN, true negative.

weaknesses result mainly from the methodological limitations of included studies, particularly with respect to inadequate reporting of withdrawals and indeterminate results and blinding of operators performing and interpreting index and reference tests. This may have resulted in overestimation of test accuracy since positive index tests could plausibly influence performance and interpretation of reference spirometry. There was also a lack of head-to-head comparisons with only two studies evaluating more than one screening test. ${ }^{22}{ }^{23}$ Indirect comparisons are potentially biased because of differences in population and study characteristics.

The criteria for airflow obstruction used in the included studies is also a point of contention given that using a fixed cut-off of $\mathrm{FEV}_{1} / \mathrm{FVC}<0.7$ may lead to overdiagnosis of the elderly. ${ }^{35}{ }^{35}$ Future studies should therefore consider using a definition that accounts for age, sex and ethnicity biases, ideally using an $\mathrm{FEV}_{1} / \mathrm{FVC}$ ratio below the lower limit of normal ${ }^{36}$ and using the fixed ratio for sensitivity analyses.

Finally, the included studies did not report acceptability and uptake of screening tests, which are all important for evaluating their overall effectiveness. This review can therefore only be used to comment on test accuracy and not on comparative clinical and cost-effectiveness in routine practice, which ideally should be evaluated through head-to-head trials.

\section{Implications for research and practice}

Our findings suggest that handheld flow meters are likely to be more accurate than questionnaires for COPD screening in primary care. However, we also highlight several key limitations of previous studies. Future studies should provide clear descriptions of withdrawals, including participant flow diagrams, ensure that spirometry is performed without prior knowledge of index tests, and that indeterminate results, particularly with respect to spirometry, are reported. Future studies should also aim to recruit participants with no prior diagnosis of COPD (thus reducing the risk of spectrum bias ${ }^{37}$ ) and use a clinical case definition, rather than just airway obstruction, in order to increase generalisability to real-life practice. More studies are needed to evaluate the accuracy and effectiveness of combining screening tests and to assess their cost-effectiveness. Finally, it remains unclear whether early detection of COPD significantly improves clinical outcomes and quality of life. This should first be demonstrated in prospective studies before firm recommendations are made.

\section{CONCLUSIONS}

Handheld flow meters used under the supervision of a trained health professional are more accurate than the CDQ for detecting spirometry-confirmed COPD in 
Figure 5 Test accuracy of each screening test in a hypothetical population of 100 participants, 5 of whom have undiagnosed COPD. COPD, chronic obstructive pulmonary disease; CDQ, COPD Diagnostic Questionnaire (score threshold).
$\operatorname{CDQ}(219.5)$

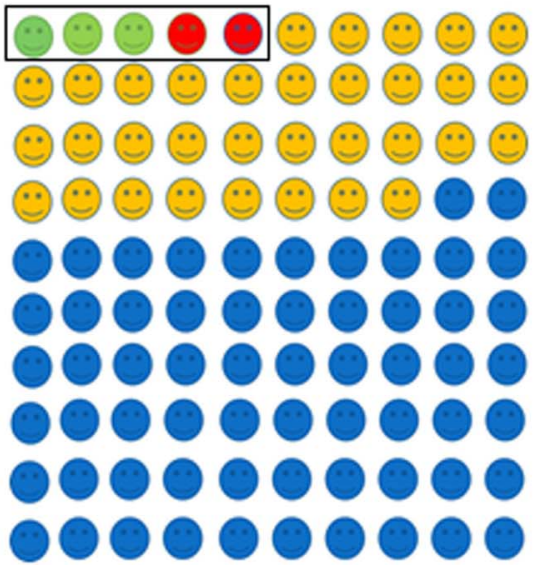

Handheld flow meter

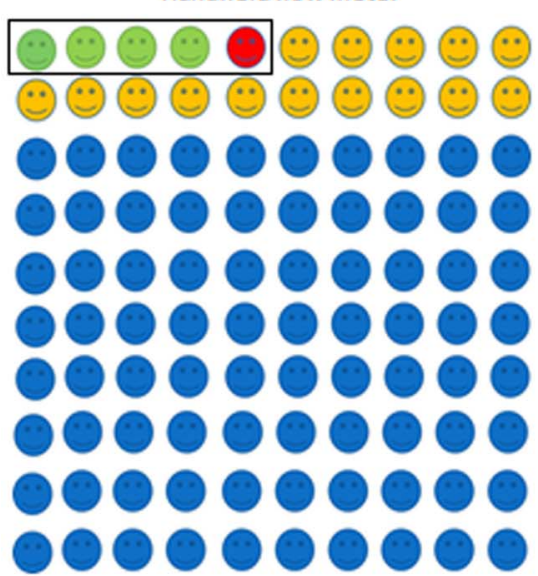

Patient with COPD appropriately referred for diagnostic assessment (true +ve)

Patient with COPD but not referred (false -ve)
$\operatorname{CDQ}(216.5)$

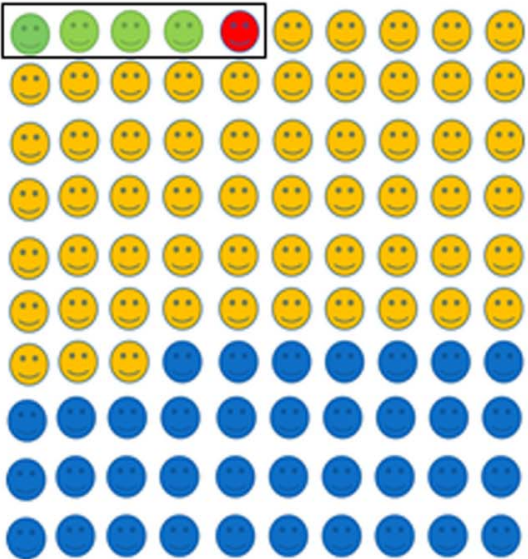

Handheld flow meter \& CDO

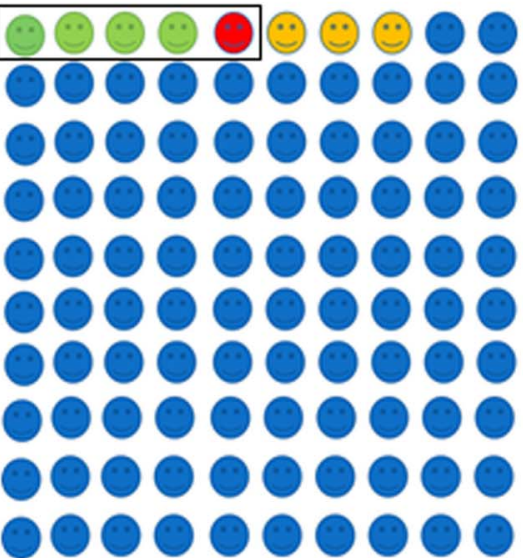

Patient without COPD referred

unnecessarily (false +ve)

Patient without COPD and not referred (true -ve) primary care. Limited evidence suggests that combining both tests may potentially improve test accuracy. Future studies should employ a case definition of COPD that aligns with current recommendations and include head-to-head comparisons.

Acknowledgements The authors would like to thank David Moore and Clare Davenport for their advice on the review protocol, Anne Fry-Smith for advice on the search strategy, Susan Bayliss (all from the University of Birmingham) for article translation, and Richard Riley (Keele University) for his advice on the statistical analysis.

Contributors SH designed the protocol, performed the literature search, selected articles, extracted data, assessed the quality of articles, synthesised the results and wrote the manuscript. RJ and PA identified the need and conceived the idea for a systematic review. RJ advised on the protocol, selected articles, verified the extracted data, assessed the quality of articles, advised on the data synthesis and revised the manuscript. YT synthesised the results (meta-analysis), advised on the risk of bias assessment and revised the manuscript. PA advised on the protocol, verified the extracted data, assessed the quality of articles, advised on the data synthesis and revised the manuscript. PA is the guarantor.

Funding This paper presents independent research funded by the National Institute for Health Research (NIHR). SH is funded by an NIHR doctoral fellowship (DRF-2011-04-064). RJ was funded by an NIHR postdoctoral fellowship (pdf/01/2008/023). YT is funded by an NIHR doctoral fellowship (DRF-2011-04-135).

Competing interests PA and RJ are both principal investigators on an NIHR programme grant for investigating COPD in primary care (grant reference number RP-PG-0109-10061).

Provenance and peer review Not commissioned; externally peer reviewed.

Data sharing statement No additional data are available.

Open Access This is an Open Access article distributed in accordance with the terms of the Creative Commons Attribution (CC BY 4.0) license, which permits others to distribute, remix, adapt and build upon this work, for commercial use, provided the original work is properly cited. See: http:// creativecommons.org/licenses/by/4.0/

\section{REFERENCES}

1. Lozano R, Naghavi M, Foreman K, et al. Global and regional mortality from 235 causes of death for 20 age groups in 1990 and 2010: a systematic analysis for the Global Burden of Disease Study 2010. Lancet 2012;380:2095-128.

2. Murray CJ, Vos T, Lozano R, et al. Disability-adjusted life years (DALYs) for 291 diseases and injuries in 21 regions, 1990-2010: 
a systematic analysis for the Global Burden of Disease Study 2010. Lancet 2012;380:2197-223.

3. Britton M. The burden of COPD in the U.K.: results from the Confronting COPD survey. Respir Med 2003:97(Suppl C):S71-9.

4. Soriano J, Zielinski J, Price D. Screening for and early detection of chronic obstructive pulmonary disease. Lancet 2009;374: 721-32.

5. Fromer L. Diagnosing and treating COPD: understanding the challenges and finding solutions. Int J Gen Med 2011;4:729-39.

6. Jones RCM, Price D, Ryan D, et al. Opportunities to diagnose chronic obstructive pulmonary disease in routine care in the UK: a retrospective study of a clinical cohort. Lancet Respir Med 2014:2:267-76.

7. Scanlon PD, Connett JE, Waller LA, et al. Smoking cessation and lung function in mild-to-moderate chronic obstructive pulmonary disease. The Lung Health Study. Am J Respir Crit Care Med 2000;161(2 Pt 1):381-90.

8. Chronic obstructive pulmonary disease: management of chronic obstructive pulmonary disease in adults in primary and secondary care. London: National Institute for Health and Clinical Excellence, 2010.

9. Jordan R, Lam KB, Cheng K, et al. Case finding for chronic obstructive pulmonary disease: a model for optimizing a targeted approach. Thorax 2010;65:492-8.

10. An outcomes strategy for people with chronic obstructive pulmonary disease (COPD) and asthma in England. London: Department of Health, 2011

11. Global initiative for chronic obstructive lung disease: global strategy for the diagnosis, management, and prevention of chronic obstructive pulmonary disease. Global Initiative for Chronic Obstructive Lung Disease, 2013.

12. Lin K, Watkins B, Johnson T, et al. Force USPST. Screening for chronic obstructive pulmonary disease using spirometry: summary of the evidence for the U.S. Preventive Services Task Force. Ann Intern Med 2008;148:535-43.

13. Kotz D, Nelemans $P$, van Schayck CP, et al. External validation of a COPD diagnostic questionnaire. Eur Respir J 2008;31:298-303.

14. Thorn J, Tilling B, Lisspers $\mathrm{K}$, et al. Improved prediction of COPD in at-risk patients using lung function pre-screening in primary care: a real-life study and cost-effectiveness analysis. Prim Care Respir $J$ 2012;21:159-66.

15. van Schayck CP, Halbert RJ, Nordyke RJ, et al. Comparison of existing symptom-based questionnaires for identifying COPD in the general practice setting. Respirology 2005;10:323-33.

16. Haroon SM, Adab PA, Jordan RE. Case finding for COPD in primary care: a systematic review. Prim Care Respir J 2012;21:354-7.

17. PROSPERO. International prospective register of systematic reviews. New York: Centre for Reviews and Dissemination, University of York, 2014.

18. Whiting $P$, Rutjes AW, Dinnes J, et al. Development and validation of methods for assessing the quality of diagnostic accuracy studies. Health Technol Assess 2004;8: iii, 1-234.

19. Reitsma JB, Glas AS, Rutjes AW, et al. Bivariate analysis of sensitivity and specificity produces informative summary measures in diagnostic reviews. J Clin Epidemiol 2005;58: 982-90.
20. Chu H, Cole SR. Bivariate meta-analysis of sensitivity and specificity with sparse data: a generalized linear mixed model approach. $J$ Clin Epidemiol 2006;59:1331-2; author reply 2-3.

21. Takwoingi Y, Guo B, Riley RD, et al. Performance of methods for meta-analysis of diagnostic test accuracy with few studies or sparse data. Stat Methods Med Res 2015

22. Sichletidis L, Spyratos D, Papaioannou M, et al. A combination of the IPAG questionnaire and PiKo-6(R) flow meter is a valuable screening tool for COPD in the primary care setting. Prim Care Respir J 2011;20:184-9, 1 p following 9.

23. Frith P, Crockett A, Beilby J, et al. Simplified COPD screening: validation of the PiKo-6(R) in primary care. Prim Care Respir $J$ 2011;20:190-8, 2 p following 8.

24. Mintz ML, Yawn BP, Mannino DM, et al. Prevalence of airway obstruction assessed by lung function questionnaire. Mayo Clin Proc 2011;86:375-81.

25. Buffels J, Degryse J, Heyrman J, et al. Office spirometry significantly improves early detection of COPD in general practice: the DIDASCO Study. Chest 2004;125:1394-9.

26. Duong-Quy S, Hua-Huy T, Mai-Huu-Thanh B, et al. [Early detection of smoking related chronic obstructive pulmonary disease in Vietnam]. Rev Mal Respir 2009;26:267-74.

27. Hanania NA, Mannino DM, Yawn BP, et al. Predicting risk of airflow obstruction in primary care: validation of the lung function questionnaire (LFQ). Respir Med 2010;104:1160-70.

28. Price DB, Tinkelman DG, Nordyke RJ, et al. Scoring system and clinical application of COPD diagnostic questionnaires. Chest 2006;129:1531-9.

29. Cartwright S. An evaluation of screening for COPD against the National Screening Committee criteria, 2012. Public Health England. http://www.screening.nhs.uk/copd

30. The UK NSC policy on chronic obstructive pulmonary disease. Public Health England, UK National Screening Committee, 2013.

31. Jing JY, Huang TC, Cui W, et al. Should fev1/fev6 replace fev1/fvc ratio to detect airway obstruction? A metaanalysis. Chest 2009;135:991-8.

32. Stanley AJ, Hasan I, Crockett AJ, et al. Validation of the COPD Diagnostic Questionnaire in an Australian general practice cohort: a cross-sectional study. Prim Care Respir J 2014;23:92-7.

33. van den Bemt L, Wouters BCW, Grootens J, et al. Diagnostic accuracy of pre-bronchodilator FEV1/FEV6 from microspirometry to detect airflow obstruction in primary care: a randomised cross-sectional study. NPJ Prim Care Respir Med 2014;24.

34. Hardie JA, Buist AS, Vollmer WM, et al. Risk of over-diagnosis of COPD in asymptomatic elderly never-smokers. Eur Respir J 2002;20:1117-22.

35. Miller MR, Levy ML. Chronic obstructive pulmonary disease: missed diagnosis versus misdiagnosis. BMJ 2015;351:h3021.

36. Quanjer P, Stanojevic S, Cole T, et al. Multi-ethnic reference values for spirometry for the 3-95-yr age range: the global lung function 2012 equations. Eur Respir J 2012;40:1324-43.

37. Ransohoff DF, Feinstein AR. Problems of spectrum and bias in evaluating the efficacy of diagnostic tests. $N$ Engl J Med 1978;299:926-30.

38. Freeman D, Nordyke RJ, Isonaka S, et al. Questions for COPD diagnostic screening in a primary care setting. Respir Med 2005;99:1311-18. 